\title{
Aplicación de metodologías activas para mejora del aprendizaje y desarrollo de competencias transversales. Experiencia en una asignatura de calidad de aguas de máster universitario.
}

\section{Carmen Hernández-Crespo ${ }^{a}$, Miguel Martín ${ }^{\mathrm{b}}$ y Javier Paredes}

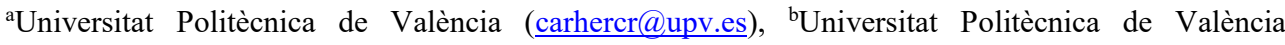
(mmartin@hma.upv.es) ycUniversitat Politècnica de València (jparedea@hma.upv.es).

\begin{abstract}
Integration in the European Higher Education Area requires the implementation of active learning methodologies that focus on the student and promote the development of transversal competences. In the present study the experience carried out in a subject of University Master is exposed. The active methodologies applied are the resolution of practical cases and the field visit where various activities are carried out to recognize in situ the knowledge acquired in the classroom. The results of a specific survey completed by the students at the end of the course are very positive, indicating a good acceptance of the applied methodologies. The grades obtained by the students in the evaluation activities also show that the learning has been deep and that different transversal competences have been adequately worked.
\end{abstract}

Keywords: Active learning methodologies, transversal competences, mathematical modeling, water quality, wetlands

\begin{abstract}
Resumen
La integración en el Espacio Europeo de Educación Superior requiere la implementación de metodologías activas de aprendizje, que centren el protagonismo en el alumno y fomenten el desarrollo de competencias transversales. En el presente estudio se expone la experiencia llevada a cabo en una asignatura de Máster Universitario. Las metodologías activas aplicadas son la resolución de casos prácticos y la visita de campo durante la que se realizan diversas actividades para reconocer in situ los conocimientos adquiridos en el aula. Los resultados de una encuesta especifica cumplimentada por los estudiantes al finalizar el curso son muy positivos, indicando una buena aceptación de las metodologías aplicadas. Las calificaciones obtenidas por los alumnos en las actividades de evaluación también muestran que el aprendizaje ha sido profundo y que se han trabajado adecuadamente diversas competencias transversales.
\end{abstract}

Palabras clave: metdologías activas de aprendizaje, competencias transversales, modelación matemática, calidad del agua, humedales. 


\section{Introducción}

El Espacio Europeo de Educación Superior (EEES) nace en 1988 con el objetivo fundamental de armonizar los sistemas de educación superior de los estados miembros, fomentando la creación de empleo y favoreciendo la movilidad de estudiantes, profesores y personal administrativo de las universidades (www.eees.es), así como ayudar a mejorar la empleabilidad de los titulados y la movilidad laboral.

El reciente informe de seguimiento del Parlamento Europeo (2015/2039(INI)) destaca que las reformas de Bolonia han posibilitado la consecución de un avance importante en cuanto a convergencia estructural de las estructuras de educación superior, pero pone de relieve la brecha todavía existente en la adaptación de los sistemas educativos a las necesidades del mercado laboral, con el fin de mejorar la empleabilidad y la competitividad. El concepto de empleabilidad implica que los estudiantes deben dominar una amplia gama de diversas competencias, para lo cual se alienta a la cooperación nacional e internacional entre la comunidad universitaria y las empresas. Así mismo, el informe pone énfasis en la necesidad de un cambio de paradigma educativo, hacia un enfoque centrado en el estudiante, que contemple su desarrollo personal, destacando la importancia de su participación en la gobernanza del sistema de educación superior.

Por tanto, entre los compromisos adquiridos en este nuevo marco de educación se encuentra el de fomentar el aprendizaje activo por parte de los estudiantes. El aprendizaje activo centra el protagonismo del proceso en el alumno, quien participa activamente en la creación de significado, es autónomo, auto-regulado y conocedor de sus propios procesos cognitivos. y el papel del docente es el de mediador entre el conocimiento y el alumnado (Olmedo, 2013). Además, las metodologías activas favorecen la formación en determinadas competencias transversales y permiten el acercamiento del estudiante a su futura labor profesional (Sancho et al., 2016).

La eficacia de las metodologías activas se puede evaluar por diferentes vías, como son las encuestas de satisfacción o la mención en el portafolio de la asignatura (Santiago, 2016), la comparación de las calificaciones obtenidas por los alumnos en años anteriores y posteriores a la implementación de la técnica objeto de estudio (Trigueros et al. 2016), o mediante la comparación de resultados de encuestas de conocimientos pasadas al inicio y al final del curso (Sancho et al., 2016).

\section{Objetivos}

El presente estudio tiene por objetivos compartir la experiencia en la aplicación de metodologías activas de aprendizaje desarrollada en una asignatura de máster universitario, así como evaluar el resultado de la experiencia a través de la consulta a los estudiantes. Por su parte, la aplicación de las metodologías seleccionadas, trabajo de casos prácticos y visita de campo, tenían por finalidad fomentar el aprendizaje activo y profundo por parte de los estudiantes así como la puesta en práctica de muchas de las competencias transversales definidas en la UPV, principalmente: la aplicación y el pensamiento práctico; el análisis y

(cc) EY-NC-ND 2017, Universitat Politècnica de València 
la resolución de problemas; la innovación y la creatividad; el trabajo en equipo y liderazgo; la responsabilidad ética, medioambiental y profesional; la comunicación efectiva; el pensamiento crítico; el conocimiento de problemas contemporáneos; el aprendizaje permanente; y la planificación y gestión del tiempo.

\section{Desarrollo de la innovación}

La experiencia descrita en la presente comunicación viene aplicándose desde el primer curso de impartición del Máster de Ingeniería Hidráulica y Medio Ambiente, concretamente en la asignatura de Modelación matemática de calidad de aguas, habiéndose observado buenos resultados en cuanto al aprendizaje adquirido por los alumnos y buena opinión por parte de los estudiantes, a través de las encuestas realizadas por el Instituto de Ciencias de la Educación de la UPV. La novedad aplicada el pasado curso reside en la introducción de una nueva metodología activa, la visita de campo, y la evaluación específica de ambas metodologías mediante una encuesta de satisfacción creada al efecto y pasada a los alumnos al finalizar el curso.

Las dos metodologías activas aplicadas son: a) la resolución de un caso práctico mediante trabajo en equipos de dos personas y b) una visita de campo a un ecosistema acuático donde se dan los problemas de contaminación vistos en la asignatura.

a) Caso práctico.

La resolución del caso práctico consiste en la implementación de un modelo matemático para evaluar la evolución de la calidad del agua de una masa acuática, comprobar si cumple los objetivos de calidad y en caso contrario proponer medidas para recuperar su buen estado, las cuales deben ser comprobadas previamente mediante simulación en el modelo desarrollado. Hacia mediados de cuatrimestre se convoca a todos los grupos a una presentación parcial del trabajo, para controlar que van progresando adecuadamente en la implementación del modelo y en la búsqueda de la información necesaria. Otro aspecto positivo es que es un buen momento para resolver las dudas que les han ido surgiendo hasta el momento. Esta entrega parcial es evaluada y forma parte de la nota final, con el fin de que se preocupen por avanzar el trabajo debidamente. Al final del curso, el trabajo completo es presentado oralmente y mediante un informe escrito, acompañado del correspondiente archivo del modelo. Ambas presentaciones, parcial y final, son en formato reunión con los profesores, quienes van formulando preguntas y comentarios a medida que se va avanzando en la presentación. Consideramos que esta modalidad es más eficiente que una presentación oral unidireccional ante toda la clase, ya que permite al estudiante sentirse más cómodo, y reflexionar y dialogar más tranquilamente con el profesor durante toda la reunión.

A lo largo del curso, los estudiantes trabajan las competencias específicas de la asignatura, como la implementación y resolución de ecuaciones diferenciales, establecimiento de cinéticas para los diferentes procesos, búsqueda de información relativa a los contaminantes a modelar (fuentes y comportamiento en la naturaleza principalmente), algunas de las cuales están estrechamente relacionadas con diversas competencias transversales. En la 
Tabla 1 se recogen las tareas desarrolladas a lo largo del caso práctico y las competencias transversales con las que se relacionan.

Tabla 1. Tareas enmarcacadas en el caso práctico y competencias transversales relacionadas.

\begin{tabular}{|c|c|}
\hline Tareas & Competencias transversal \\
\hline $\begin{array}{l}\text { Búsqueda de información sobre los contaminantes } \\
\text { a modelar. }\end{array}$ & CT-01, CT-0 \\
\hline $\begin{array}{l}\text { Implementación del modelo matemático y } \\
\text { resolución. }\end{array}$ & $\begin{array}{l}\text { CT-01; CT-02; CT-03; CT-05; CT-06; CT- } \\
12 ; \text { CT-13. }\end{array}$ \\
\hline $\begin{array}{l}\text { Búsqueda de normative aplicable y comprobación } \\
\text { de su cumplimiento. }\end{array}$ & CT-01; CT-06; СТ-07; CT-10. \\
\hline $\begin{array}{l}\text { Simulación de medidas para recuperar el buen } \\
\text { estado de la masa de agua. }\end{array}$ & $\begin{array}{l}\text { CT-01; CT-02; CT-03; CT-04; CT-05; CT- } \\
\text { 06; CT-07; CT-09; CT-11; CT-12 }\end{array}$ \\
\hline $\begin{array}{l}\text { Exposición del caso mediante presentación oral e } \\
\text { informe escrito. }\end{array}$ & $\begin{array}{l}\text { CT-01; CT-02; CT-04; CT-06; CT-07; CT- } \\
08 ; \text { CT-09; CT-12. }\end{array}$ \\
\hline \multicolumn{2}{|c|}{$\begin{array}{l}\text { CT-01. Comprensión e integración; CT-02. Aplicación y pensamiento práctico; CT-03. Análisis y } \\
\text { resolución de problemas; CT-04. Innovación, creatividad y emprendimiento; CT-05. Diseño y proyecto; } \\
\text { CT-06. Trabajo en equipo y liderazgo; CT-07. Responsabilidad ética, medioambiental y profesional; CT- } 08 \text {. } \\
\text { Comunicación efectiva; CT-09. Pensamiento crítico; CT-10. Conocimiento de problemas contemporáneos; } \\
\text { CT-11. Aprendizaje permanente; CT-12. Planificación y gestión del tiempo; CT-13. Instrumental específica. }\end{array}$} \\
\hline
\end{tabular}

b) Visita de campo.

La visita de campo se realizó a una masa de agua que sufre algunos de los problemas de contaminación del agua explicados en la asignatura, l'Albufera de València. Concretamente dentro del Parque Natural de la Albufera visitamos el área de reserva del Tancat de la Pipa, el cual dispone de unas zonas de humedal artificial como sistema de tratamiento del agua procedente del lago seguido de unas lagunas someras.

En el Tancat de la Pipa pudieron conocer, de la mano de los técnicos de gestión de la reserva, la historia sobre el proceso de formación de l'Albufera y el proceso de deterioro ambiental que sufrió a partir de la década de los 60, así como las medidas que hay actualmente en marcha para revertir su estado. Posteriormente se realizó un recorrido a lo largo del Tancat y fueron identificando, con ayuda del profesor, las diferencias entre los distintos ambientes creados en el área de reserva, así como proponiendo formas de introducir los distintos procesos y variables observadas in situ en los modelos matemáticos. También ser realizó un ejercicio de tomar muestra en la entrada del sistema (procedente del lago de la Albufera) y salida del mismo, observando las grandes diferencias entre las mismas y poniendo de manifiesto el buen funcionamiento del mismo en cuanto a mejora de la calidad de las aguas.

De forma resumida, los principales procesos que se identificaron fueron los de sedimentación y resuspensión de partículas, los aportes por parte de la fauna (peces y aves), los procesos de degradación de materia orgánica, la eutrofización del agua procedente del lago, la asimilación de nutrientes por parte de la vegetación helófita. Las principales 
variables a modelar observadas fueron: la materia orgánica, el fitoplancton, la vegetación, las aves y la ictiofauna.

En definitiva, los humedales son espacios de gran valor educativo, caracterizados por ofrecer una amplia variedad de funciones y valores, tanto ambientales como sociales, económicos y culturales, por lo que representan áreas adecuadas para el desarrollo de actividades con enfoque holístico, integrando diferentes áreas de conocimiento (hidrología, calidad del agua, biología, geomorfología, ordenación del territorio, etc) (García et al. 2012).

Tras finalizar el recorrido por el Tancat de la Pipa, nos recogió una barca que nos trasladó al embarcadero de la Gola de Puchol atravesando el lago, de manera que pudieron comprobar los problemas de eutrofización que éste sufre y conocer un poco más la idiosincrasia del entorno gracias a las anécdotas contadas por el barquero. En la Figura 1 se muestran algunas imágenes de la visita realizada.
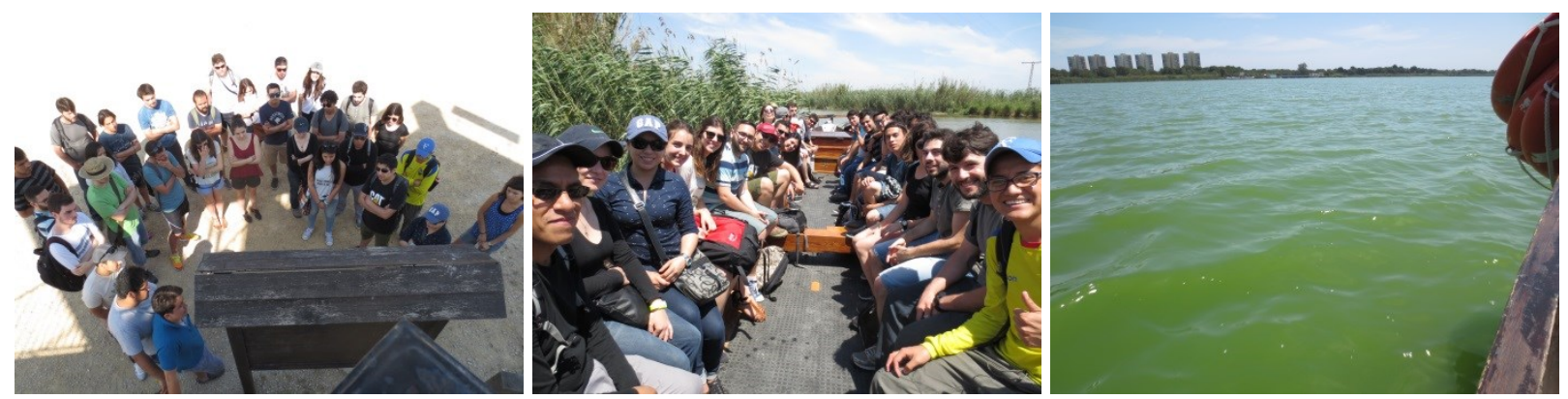

Figura 1. Imágenes de la visia al Tancat de la Pipa en l'Albufera de València.

\section{Resultados}

Los resultados de la aplicación de metodologías activas fueron satisfactorios, los alumnos demostraron haber adquirido un aprendizaje profundo de la materia, siendo capaces de resolver los casos prácticos de manera adecuada, algunos incluso con simulaciones muy creativas y en general unas presentaciones orales estructuradas y bien explicadas. Las calificaciones obtenidas fueron muy buenas, obteniendo todos los grupos entre notable (34\% de los grupos) y sobresaliente (66\% de los grupos).

La visita no fue evaluada y calificada pero sí se preguntó a los asistentes si les había parecido una actividad interesante, que les acercaba del papel y el ordenador a la realidad, y la respuesta fue positiva. Los autores consideran que para poder modelar bien un problema es preciso conocerlo personalmente, visitando el lugar, ya que te permite conocer detalles que son difícilmente detectables a través del análisis de la información numérica.

Como se comentaba en la introducción, existen diversas vías para medir la eficacia de las metodologías empleadas. En nuestro caso, la impresión que tuvieron los docentes al hacer la evaluación de los trabajos realizados fue buena, se comprobó que los estudiantes habían comprendido la materia y eran capaces de explicar adecuadamente las tareas desarrolladas 
en el trabajo. Por otro lado, se deseaba conocer la percepción de los estudiantes sobre las técnicas de aprendizaje aplicadas, para lo cual se elaboró una encuesta de satisfacción anónima que fue respondida por los alumnos $(\mathrm{N}=20)$.

En la encuesta se formularon seis preguntas de satisfacción y una de selección de competencias transversales que consideran haber desarrollado durante la asignatura. Las preguntas seguían la escala de valoración de las encuestas realizadas por el ICE, con la que ya estaban familiarizados los estudiantes, esto es: 1) totalmente en desacuerdo (valoración muy negativa), 2) más bien en desacuerdo, 3) término medio, 4) más bien de acuerdo, 5) totalmente de acuerdo (valoración muy positiva). A continuación se datallan las preguntas realizadas:

1. El sistema de evaluación de la asignatura, con puntuación distribuida entre exámenes y trabajos, me ha parecido adecuado.

2. El trabajo de la asignatura, basado en la resolución de un caso práctico, me ha parecido interesante y de utilidad para reforzar los conocimientos adquiridos en la asignatura.

3. La revisión parcial del trabajo con el profesor me parece una tarea importante porque me ha ayudado a detectar errores y poder corregirlos, plantear dudas y obligarme a avanzar una parte del trabajo y no dejarlo todo para el final.

4. La presentación final del trabajo en un formato de reunión, con diálogo con el profesor me ha servido para sentirme cómodo/a a la hora de expresarme y explicar el trabajo realizado. Lo prefiero respecto a presentación oral unidireccional.

5. La visita de campo de la asignatura me ha permitido reconocer en el mundo real los procesos y problemas de contaminación que hemos aprendido a modelar en la asignatura.
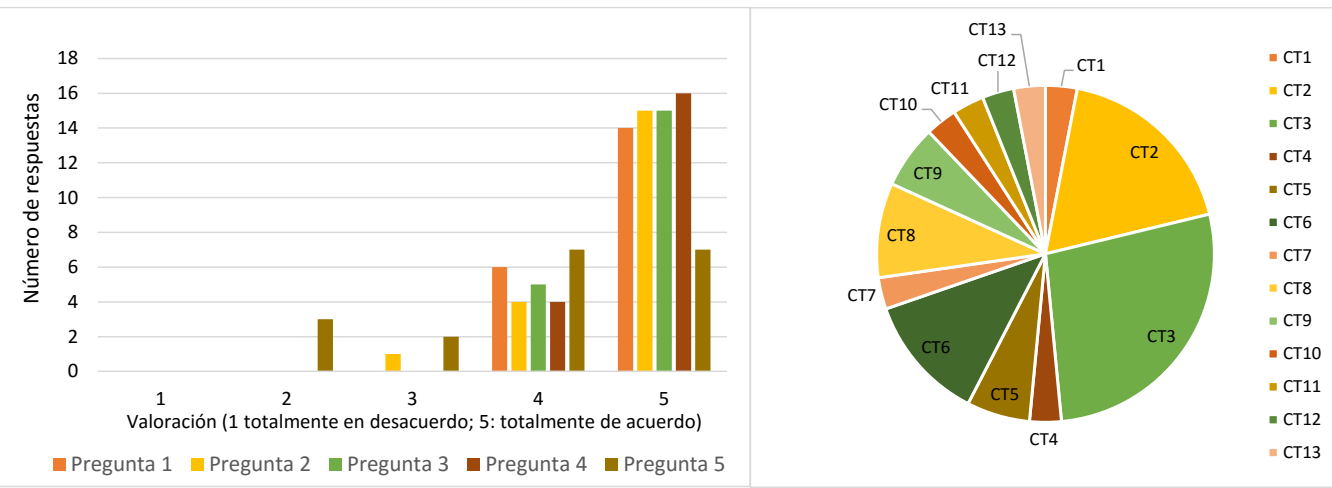

Figura 2. Resultados de la encuesta de valoración de las metodologías activas realizadas. Izda: histograma de las respuestas a las distintas preguntas realizadas. Dcha: competencias transversales desarrolladas por los alumnos.

Los resultados de la valoración fueron muy positivos, ya que la mayor parte de los encuestados calificaron con la puntuación máxima las cinco preguntas (Figura 2).

(c) EY-NC-ND 2017, Universitat Politècnica de València 
Por otro lado, otra de las preguntas consistía en que el estudiante seleccionase aquellas competencias transversales que consideraba haber desarrollado a lo largo de la asignatura. Las que en mayor medida se identificaron fueron la CT2 - aplicación y pensamiento práctico (18\%), CT3 - análisis y resolución de problemas (27\%), CT6 - trabajo en equipo y liderazgo (12\%) y CT8 - Comunicación efectiva (9\%). Estos resultados nos indican que sí que fueron perfectamente reconocidas aquellas más explícitas, pero que debemos poner mayor énfasis en mostrar cómo las actividades desarrolladas contribuyen a poner en práctica prácticamente todo el conjunto de competencias transversales definidas en la UPV.

Así mismo, se les preguntó si el momento de la visita, que en este curso se hizo al final del curso, les había parecido adecuado. La respuesta fue positiva por parte del $58 \%$ de los alumnos, mientras que el resto habría preferido a mitad de curso (26\%) o al inicio del curso $(16 \%)$. Esta valoración será tenida en cuenta de cara a la programación de la visita para el siguiente curso, ya que es posible que hacia mitad del curso se le pueda sacar un mayor partido y sirva como referencia para los siguientes temas a impartir, así como para comprender mejor algunos de los aspectos del caso práctico.

\section{Conclusiones}

La principal conclusión extraída del estudio es que las metodologías activas de aprendizaje fomentan un aprendizaje más profundo, centrado en el estudiante, viéndose reflejado en las actividades de evaluación y en las calificaciones. Además, el estudiante es capaz de reconocer la eficacia de las metodologías, valorándolas muy positivamente, e identificar las principales competencias transversales que han trabajado durante la asignatura.

\section{Referencias}

García Fernández, B., Sánchez Emeterio, G., Sánchez Ramos, D. (2012). La transposición didáctica de la investigación en humedales. Estudios sobre el Mensaje Periodístico, 18, 347-356. http://dx.doi.org/10.5209/rev ESMP.2012.v18.40988.

Olmedo Moreno, E. M. (2013). Enfoques de aprendizaje de los estudiantes y metodología docente: Evolución hacia el nuevo sistema de formación e interacción propuesta en el EEES. Revista de Investigación Educativa, 31 (2), 411-429. http://dx.doi.org/10.6018/rie.31.2.133501.

Parlamento Europeo. 2015/2039(INI). Informe sobre el seguimiento de la aplicación del Proceso de Bolonia. Comisión de cultura y educación.

Sancho Fernández, M., García-Fayos, B. y Arnal Arnal, M. (2016). "Análisis de la mejora del aprendizaje tras la incorporación de metodologías activas en una asignatura optativa del Grado de Ingeniería Química” en Congreso Nacional de Innovación Educativa y de Docencia en Red. Editorial Universitat Politècnica de València. http://dx.doi.org/10.4995/INRED2016.2016.4407.

Santiago Martín de Madrid, P. (2016). "La motivación del alumnado derivada de la realización de prácticas en contextos reales" en en Congreso Nacional de Innovación Educativa y de Docencia en Red. Editorial Universitat Politècnica de València. http://dx.doi.org/10.4995/INRED2016.2016.4375. 
Trigueros Preciado, S., Pérez González, D., Solana González, P., García Ruiz, E. (2016). "Experiencia docente universitaria mediante el uso de metodologías activas para el aprendizaje como la evaluación formativa compartida y el trabajo de competencias trasnversales" en Congreso Nacional de Innovación Educativa y de Docencia en Red. Editorial Universitat Politècnica de València. http://dx.doi.org/10.4995/INRED2016.2016.4351.

2017, Universitat Politècnica de València 